\title{
Spectroscopy and Quantum-Dynamics: From Vibrations to Reactions
}

\author{
David Luckhaus* \\ Werner Prize Winner 2001
}

\begin{abstract}
The physical understanding of chemical reactivity builds on the connection between structural and dynamical molecular properties, such as the specific dynamical properties of functional groups which determine a molecule's chemical behavior. Studies of $\mathrm{OH}-$ and $\mathrm{NH}_{2}$ groups in different environments demonstrate how experiment and theory combine to draw a detailed picture of the molecular quantumdynamics. The hydrogen motion in a series of model systems elucidates important aspects of the N/O/H chemistry, with implications for radical and atmospheric chemistry. In perfect analogy to the separation of electronic and nuclear motion in the Born-Oppenheimer approximation, characteristic motions of individual structural features are adiabatically separated from the overall system dynamics. This phenomenon of vibrational adiabaticity will play a central role in the understanding of the microscopic foundations of empirical structure-reactivity relationships.
\end{abstract}

Keywords: Molecular quantum dynamics · Spectroscopy · Structure-reactivity relationship · Vibrational adiabaticity

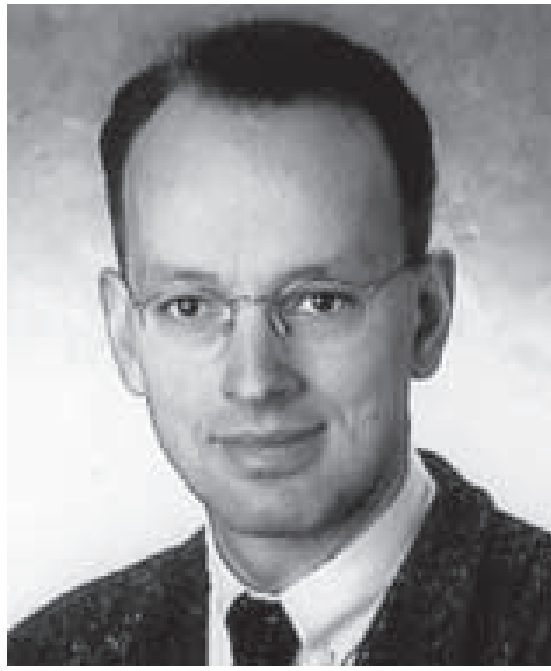

${ }^{*}$ Correspondence: PD Dr. D. Luckhaus

Laboratorium für Physikalische Chemie ETH Zürich

ETH-Hönggerberg HCl

$\mathrm{CH}-8093$ Zurich

Present address:

Institut für Physikalische Chemie

Universität Göttingen

Tammannstrasse 6

D-37077Göttingen

Tel.: +49551393126

Fax: +49551393117

E-Mail: dluckha@gwdg.de
David Luckhaus, born 1961 in Remscheid (Germany), studied chemistry at the universities of Cologne and Göttingen. He received his diploma in chemistry in 1987 from the University of Göttingen with the thesis 'The Optimization of Molecular Geometries in Electronically Excited States within the CNDO-Approximation'. Working on high-resolution IR spectroscopy with Prof. Martin Quack at ETH Zürich he received his Dr. sc. nat. in 1991 with a thesis on 'Spectroscopic and Theoretical Investigations of the Vibrational Dynamics of Dideuteromethylfluoride'.

David Luckhaus was postdoctoral fellow at ETH Zürich (1991/2) and at the University of Wisconsin (Madison, USA) (1992/3), where he worked with Prof. F. Fleming Crim on IR/UV double resonance spectroscopy, collisional energy transfer, and vibrationally mediated photodissociation of highly excited molecules. In 1994 and 1997 he spent short periods as visiting scientist at the University of Wisconsin From 1993 to 1998 he was research assistant, senior research assistant (1997) and lecturer (1998) at the Department of Chemistry of ETH Zürich.

In 1999 he was named Privatdozent on the basis of his Habilitationsschrift 'Molecular Quantum Dynamics and Spectroscopy'. In 2000 David Luckhaus was awarded the Latsis Prize of ETHZürich and the Akademiepreis für Chemie der Akademie der Wissenschaften zu Göttingen. Current research interests are centered around the investigation of the microscopic foundations of chemical reactivity combining experimental and theoretical methods of modern molecular spectroscopy.

\section{Introduction}

Spectroscopy and quantum dynamics what do they have to do with chemistry? What can the interaction of matter with light tell us about the fast processes on a molecular scale that are chemical reactions? The purpose of this article is to illustrate how the proper combination of experiment and theory can translate highresolution spectra into a detailed picture of the molecular quantum dynamics that induce chemical change.

Unraveling the mechanisms of energy transfer on a molecular level is one of the central problems of chemical reaction kinetics. Most intriguing from the chemist's point of view is the connection between a molecule's dynamical and structural properties. Although empirically well-established, this relationship leaves many open questions. Which are its microscopic foundations? Are there transferable properties of functional groups and how do they determine the course of chemical reactions? Modern spectroscopy opens a unique approach to these problems. The key is provided by the interpretation of molecular spectra in terms 
of explicit quantum-mechanical models of the underlying molecular motion.

\section{Experimental Approach}

The analysis of high resolution rovibrational spectra on the basis of microscopic models of the molecular motion has been used with great success to reveal 'hidden' (approximate) symmetries, coupling hierarchies, and 'constants' of the motion on different time scales not easily detected in time-dependent experiments [1-3]. The experimental basis of such studies is provided by high-resolution spectroscopy ranging from the far-infrared up to the visible range of the spectrum, covering rotations and slow large amplitude vibrations as well as the fast energy redistribution processes in highly excited molecules. The measurements are stationary but the resolution of spectra determines the time scales accessible through this approach while the band width limits the temporal resolution. These needs together with the requirement of high sensitivity are not easily reconciled by a single experimental method. Modern spectroscopy provides a whole arsenal of techniques, with different strengths and weakness, which can be combined. So the broad band width of Fourier transform IR/Vis spectroscopy supplements the high resolution and sensitivity of various Laser spectroscopic techniques (e.g. photo-acoustic [4][5] and cavity ring-down spectroscopy [6]. Various experimental approaches are available to simplify dense and complex spectra. Thermal congestion is most efficiently removed in supersonic jet expansions by reducing effective temperatures down to $20 \mathrm{~K}$ and below [7]. Double-resonance techniques allow selected levels to be studied in a dense manifold. Prominently among these feature double resonance ionization techniques [8][9] and vibrationally mediated photo-dissociation [10] experiments.

The enormous technical progress in modern experiments provides access to spectroscopic information on an unprecedented level of detail. This requires new methods to evaluate and analyze a huge amount of data. With automated assignment procedures and efficient simulation techniques [11] high-resolution ro-vibrational spectra with their literally hundreds of thousands of lines can be analyzed up to the highest level of detail as illustrated in Fig. 1. Beyond simple data reduction this procedure allows the identification of coupling hierarchies and ef-

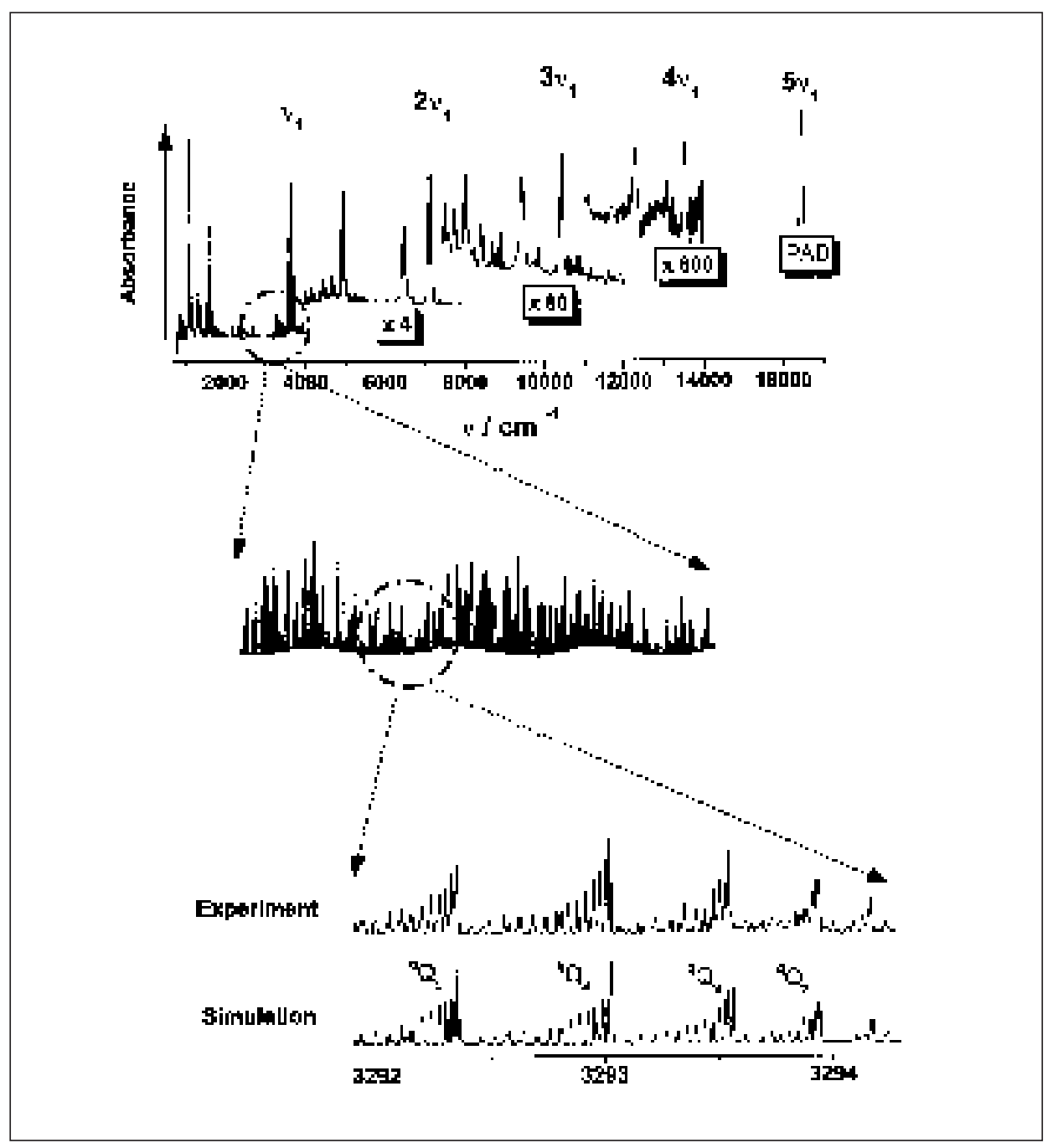

Fig. 1. The complete rotationally resolved vibrational spectrum of hydroxylamine $\left(\mathrm{NH}_{2} \mathrm{OH}\right)$. Highresolution FTIR spectra [19] except PAD: photo-acoustic overtone spectra [4].

fective symmetries. To extract the interesting dynamical information from the experimental spectroscopic data the spectra have to be simulated on the basis of a realistic model for the molecular motion.

\section{Models for the Molecular Quantum Dynamics}

The detailed modeling of the molecular quantum dynamics splits into two main problems: (i) the construction of suitable multi-dimensional potential surfaces and (ii) the solution of the equations of motion, the ro-vibrational Schrödinger equation.

Extracting potential surfaces from experimental spectra alone is wrought with difficulties. Experimental spectra only have access to a subset of the eigenvalue spectrum. But not even the complete eigenvalue spectrum allows for the unambiguous determination of a multidimensional potential function. Intensity information could provide some of the missing information but it requires knowledge of the dipole moment hypersurface. From these considerations it is clear that some a priori information is necessary to constrain any empirical determination of potential surfaces. One of the most important sources for this kind of information are quantum chemical ( $a b$ initio) calculations. Even if $a b$ initio calculations generally do not reach 'experimental accuracy' they can provide a qualitatively or even semi-quantitatively correct model for the molecular quantum dynamics. For example they are crucial for developing appropriate analytical forms for model potentials and to define 'physically reasonable' ranges for their parameterization. Ab initio theory thus provides the theoretical frame within which quantum dynamical models can be refined empirically. Numerical interpolation schemes have become increasingly efficient and versatile tools for the construction of multidimensional potential functions directly from $a b$ initio data 
[12][13] circumventing the laborious task of fitting analytical functions.

Given a potential function the next step is the solution of the ro-vibrational Schrödinger equation. In contrast to the problem of electronic motion for which various quantum chemical program packages offer numerical solutions there is no comparable approach to the numerical treatment of nuclear (ro-vibrational) quantum dynamics. Different molecules require different coordinate systems, and the Hamiltonian usually has to be rederived for each new class of systems. The recent development, however, of the Generalized Z-Matrix Discrete Variable Representation (DVR) represents significant progress towards a truly unified description of multi-dimensional molecular motion. This method is based on ideas put forward by Meyer and Günthard more than 30 years ago [14]. It relies on a very general formulation in terms of angular momenta $\left(\hat{J}_{\alpha}\right)$ and vibrational coordinates and momenta $\left(q_{j}, \hat{p}_{j}\right)$ largely independent of the coordinate system:

$$
\hat{H}=\frac{1}{2}\left(\widehat{\mathbf{J}}^{t}, \hat{\mathbf{p}}^{t}\right)\left(\begin{array}{cc}
\mathbf{I} & \mathbf{C} \\
\mathbf{C}^{t} & \mathbf{g}
\end{array}\right)^{-1}\left(\begin{array}{l}
\hat{\mathrm{J}} \\
\hat{\mathbf{p}}
\end{array}\right)+u(\mathbf{q})+V(\mathbf{q})
$$

I is the tensor of inertia, $\mathbf{C}$ is the Coriolis coupling matrix, and $\mathbf{g}$ is the covariant vibrational metric tensor, effectively the inverse reduced mass for the vibrational motion. $u(\mathbf{q})$ is a mass dependent effec- tive contribution to the potential energy $V(\mathbf{q})$. Explicit expressions in terms of the Cartesian molecular geometry can be found in [15]. It should be noted that Eqn. (1) is exact and completely general. It is particularly suitable in combination with gridbased representations of the Hamiltonian where all terms involved can be calculated numerically, without any further complicated algebra [15]. Thus one and the same computer program can be used for the complete treatment of small molecules as well as for multi-dimensional models of large systems.

\section{Vibrational Adiabaticity and Quantum Dynamics of Functional Groups}

Once the numerical tools are in place the main task still remains: the formulation of a physical-chemical model. Even if it were feasible, the complete numerical description of a complex system would not provide much insight beyond another (most probable) confirmation of quantum mechanics. The physical-chemical understanding lies in simplified models, which allow us to understand regularities, such as the separation of characteristic time scales in the motion of complex systems. One of these regularities observed in chemistry - in fact one of its building blocks - is the relation between molecular structure and reactivity. But what are the microscopic foundations of this phenomenon? Are there types of motion characteristic of certain structural features that give rise to a particular chemical behavior? Are these dynamical properties transferable and if so how are they influenced by the molecular environment? These fundamental questions can be studied by isolating the subunits of interest in small model systems where the laws of motion can be described in the greatest possible detail. But how can functional groups follow their own separate dynamics? They are bound strongly to the molecular frame, so that their motion should be strongly coupled to the latter. This seeming paradox can be resolved within an adiabatic picture in perfect analogy to the familiar Born-Oppenheimer approximation of electronic structure theory. The idea of adiabatic separability is completely general for separating fast and slow motions and has found applications in chemical kinetics [16] as well as in molecular spectroscopy [17].

\subsection{Mode-selective Stereomutation in Hydrogen Peroxide}

The mode-selective stereomutation of hydrogen peroxide $\left(\mathrm{H}_{2} \mathrm{O}_{2}\right)$ affords a perfect illustration for the effects of vibrational adiabaticity. $\mathrm{H}_{2} \mathrm{O}_{2}$ exists in two chiral equilibrium configurations along the torsional angle of the two $\mathrm{OH}$ groups with a barrier in trans conformation of about $400 \mathrm{~cm}^{-1}$. The quantum mechani-
Fig. 2. Adiabatic stereomutation tunneling in $\mathrm{H}_{2} \mathrm{O}_{2}$. Top left: adiabatic torsional potentials. Top right: Adiabatic wave packet dynamics in the predissociative continuum of the fourth $\mathrm{OH}$-stretching overtone $\left(5 \mathrm{v}_{1}\right) . \tau$ is the torsional angle. $|\Psi|^{2}$ is the time-dependent probability density in the torsional angle resulting from an exact six-dimensional wave function integrated over the remaining five coordinates. Bottom: Experimental (asterisks) and theoretical (circles) stereomutation rates as a function of the $\mathrm{OH}$ stretching overtone excitation. The $\mathrm{O}-\mathrm{O}$ dissociation limit lies around $17052 \mathrm{~cm}^{-1}$.

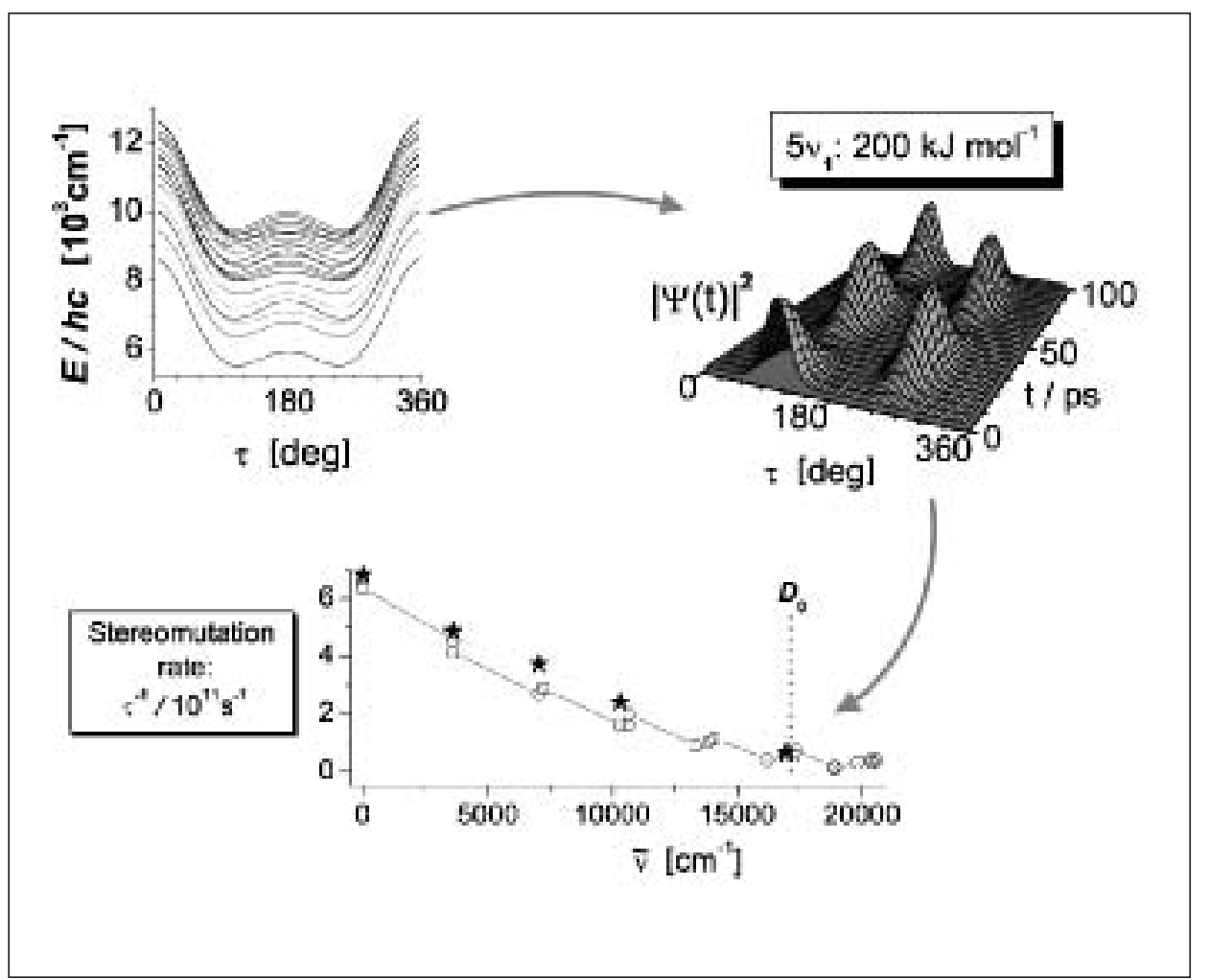


cal tunneling interconverts the two enantiomeric forms within about a picosecond even at $0 \mathrm{~K}$. In the spectrum this same effect gives rise to a small splitting of the ground state by $11 \mathrm{~cm}^{-1}$. The splittings observed in the infrared spectrum for vibrationally excited states, however, vary all over the place, sometimes tunneling is faster, sometimes it is slower than in the ground state. How can we make sense of such a behavior at energies high above the torsional barrier?

We have been able to show that this is the immediate result of the adiabaticity of the torsional stereomutation dynamics [18]. As illustrated in Fig. 2 there is a separate effective torsional potential for each state of the fast stretching and bending vibrations just like the electronic potentials of the Born-Oppenheimer approximation. Each adiabatic potential has a different torsional barrier. Exciting the asymmetric bending vibration, for example, accelerates the tunneling by reducing the effective barrier. But the opposite effect is observed, too. The $\mathrm{OH}$ stretching vibration is an example where putting energy into the molecule slows down the stereomutation. One can even excite the molecule beyond the dissociation limit of the $\mathrm{O}-\mathrm{O}$ bond and still observe very regular wave packet dynamics effectively localized in the torsional degree of freedom. Fig. 2 shows the corresponding time-dependent probability density in the torsional coordinate for a chiral wave packet. Initially localized in one of the minima the wave packet performs a very regular periodic motion over many periods without any decay. Note the enor- mous increase of the tunneling period at this extremely high excitation.

The quantum dynamical simulations agree perfectly with the systematic decrease of the tunneling or stereomutation rate observed experimentally with increasing $\mathrm{OH}$ stretching excitation. Thus adiabaticity is a by no means trivial result of the experimental observations. Even if the life time of chiral states barely reaches nanoseconds it is still intriguing to speculate about the possibility of vibrationally induced chirality in reactions such as the dissociation of $\mathrm{H}_{2} \mathrm{O}_{2}$.

\subsection{Reactive Vibrational Dynamics in Hydroxylamine}

$\mathrm{H}_{2} \mathrm{O}_{2}$ provides a beautiful example for the role of adiabaticity for mode selective dynamics, but can this concept be extended to the dynamics of chemical change? A seemingly small change illustrates the idea: substituting one of the $\mathrm{OH}$ groups of $\mathrm{H}_{2} \mathrm{O}_{2}$ with a $\mathrm{NH}_{2}$ group leads to the isoelectronic hydroxylamine. As metastable intermediate it plays an important role in the radical chemistry of oxygen and nitrogen, e.g. in atmospheric and combustion processes. For the present purposes the main interest lies in the wealth of its intramolecular dynamics illustrated in Fig. 3. The interaction between the two functional groups $\mathrm{OH}$ and $\mathrm{NH}_{2}$ opens up a new path not available in $\mathrm{H}_{2} \mathrm{O}_{2}$ : The intramolecular H-transfer between the two functional groups.

The almost complete ro-vibrational spectrum of $\mathrm{NH}_{2} \mathrm{OH}$ has been measured and analyzed from the far-infrared to the visible range of the spectrum (see Fig. 1)
[19]. This is a rare example where such a complete set of information is available. The literally tens of thousands of data provide deep insight into the intramolecular quantum dynamics of this model system, which is now understood in great detail [13]. A particularly interesting aspect concerns the overtones of the $\mathrm{OH}$ stretching vibration shown in Fig. 4. Increasing excitation leads to a slight broadening of the rotational fine structure in line with the effective increase of the density of states. At the fourth overtone, however, the fine structure suddenly vanishes altogether [10]. This effect cannot be due to dissociation as the lowest singlet N-O dissociation channel lies slightly higher in energy. Rather it must reflect the onset of a fast intramolecular process.

Detailed model calculations provide strong evidence that this process must be the intramolecular H-transfer, which leads to the isomeric ammonia oxide $\mathrm{NH}_{3} \mathrm{O}$. This molecule turns out to sit in a deep potential well bound by about $100 \mathrm{~kJ} / \mathrm{mol}$. The broad absorption features result from rather unusual highly excited states with an $\mathrm{H}$ atom still bound but orbiting almost freely about a flexible $\mathrm{NH}_{2} \mathrm{O}$ core [13][20]. Fig. 4 shows the energy profile calculated for this process ab initio.

\section{Conclusions and Outlook}

The example of the reactive vibrational dynamics of $\mathrm{NH}_{2} \mathrm{OH}$ completes the line which connects molecular vibrations to chemical reactions. The examples discussed here together with many

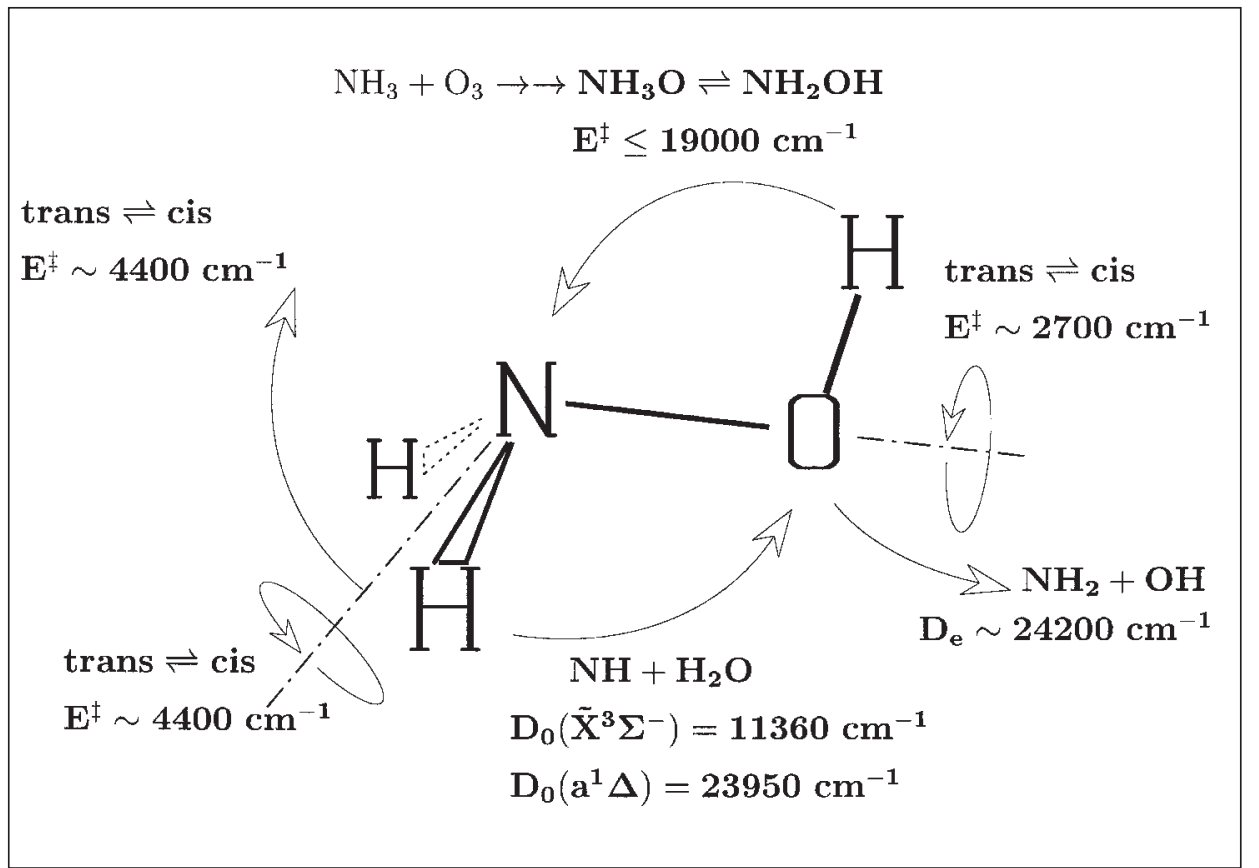

Fig. 3. Intramolecular pathways for conformational dynamics and chemical change in hydroxylamine. 


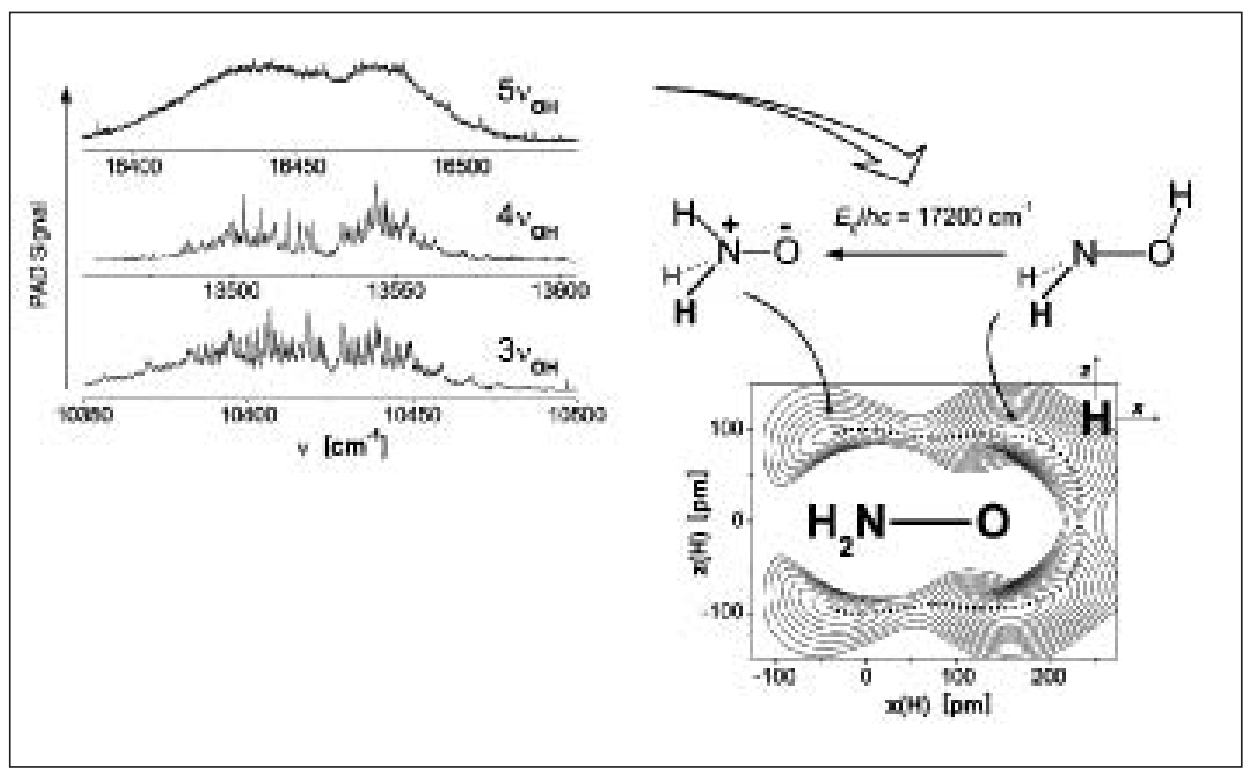

Fig. 4. Reactive vibrational dynamics in $\mathrm{NH}_{2} \mathrm{OH}$. Left: $\mathrm{OH}$ stretching overtone absorption bands. The effective ro-vibrational line widths deduced from simulations are $0.3 \mathrm{~cm}^{-1}, 0.9 \mathrm{~cm}^{-1}$, and $10 \mathrm{~cm}^{-1}$ for $3 v_{\mathrm{OH}}, 4 v_{\mathrm{OH}}$, and $5 \mathrm{v}_{\mathrm{OH}}$, respectively. Right: $2 \mathrm{D}$ minimum energy profile for the intramolecular $\mathrm{H}$-transfer in hydroxylamine. Ab initio calculations at the MP2/6-31G* level. Contour lines every $2000 \mathrm{~cm}^{-1}, \mathrm{NH}_{2} \mathrm{OH}$ minimum at $0 \mathrm{~cm}^{-1}, \mathrm{NH}_{3} \mathrm{O}$ minimum at $8409 \mathrm{~cm}^{-1}$. The saddle point energy $E_{0}$ was calculated at the $\operatorname{CCSD}(\mathrm{T}) / 6-31 \mathrm{G}^{\star *}$ level using the corresponding MP2 geometry (MP2 value: $17218 \mathrm{~cm}^{-1}$ ).

others studied in recent years provide strong evidence that the relationship between molecular structure and reactivity is determined by specific quantum-dynamical properties of individual subunits such as functional groups. Transferable properties can and do exist to the extent that the dynamics of functional groups are adiabatically separated from the overall dynamics of the molecular system. This report has concentrated on small model systems, where experiments can be compared with more detailed quantitative models and where the important dynamical features are more clear cut. But the general concept of vibrational adiabaticity has much wider scope. It is ideally suited to focus on the dynamically important aspects of complex systems. This encompasses the mode selectivity of the tunneling dynamics in large systems [17] as well as intramolecular proton transfer processes through hydrogen bonds. An interesting extension of the concept of adiabatic separation relates to the time scales of conformational change in $\mathrm{H}$ bonded networks [21].

\section{Acknowledgements}

I am indebted to Prof. M. Quack and his group at ETH Zürich for their support and encouragement. The repeated hospitality of Prof. F.F. Crim at the University of Wisconsin and the hospitality of the Sonderforschungsbereich
357 at the Universität Göttingen are gratefully acknowledged.

Received: November 20, 2001

[1] D. Luckhaus, M. Quack, Chem. Phys. Lett. 1993, 205, 277.

[2] M. Quack, in 'Femtosecond Chemistry, Proc. Berlin Conf. Femtosecond Chemistry, Berlin, March 1993', Eds J. Manz, L. Woeste, Verlag Chemie, Weinheim, 1995, Chap. 27, pp. 781-818.

[3] A. Beil, D. Luckhaus, M. Quack, Ber. Bunsenges. Phys. Chem. 1996, 100, 1853.

[4] J.L. Scott, D. Luckhaus, S.S. Brown, F.F. Crim, J. Chem. Phys. 1995, 102, 675.

[5] D. Luckhaus, M.J. Coffey, M.D. Fritz, F.F. Crim, J. Chem. Phys. 1996, 104, 3472.

[6] M. Hippler, M. Quack, Chem. Phys. Lett. 1999, 314, 273.

[7] G. Scoles, 'Atomic and Molecular Beam Methods' Oxford University Press, Oxford, 1988

[8] O.V. Boyarkin, T.R. Rizzo, J. Chem. Phys. 1995, 103, 1985.

[9] B. Fehrensen, M. Hippler, M. Quack, Chem. Phys. Lett. 1998, 298, 320.

[10] X. Luo, P.R. Fleming, T.A. Seckel, T.R. Rizzo, J. Chem. Phys. 1990, 93, 9194.

[11] D. Luckhaus, M. Quack, Mol. Phys. 1989, $68,745$.

[12] T.-K. Ha, D. Luckhaus, M. Quack, Chem. Phys. Lett. 1992, 190, 590.

[13] D. Luckhaus, Ber. Bunsenges. Phys. Chem. 1997, 101, 346.

[14] R. Meyer, H.H. Günthard, J. Chem. Phys. 1969, $50,353$.

[15] D. Luckhaus, J. Chem. Phys. 2000, 113, 1329.
[16] M. Quack, J. Troe, in 'Encyclopedia of Computational Chemistry', Eds P. Schleyer, A. Allinger, T. Clark, J. Gasteiger, P.A. Kollmann, H.F. Schaefer III, P.R. Schreiner, John Wiley \& Sons, New York, 1998, Vol. 4, pp. 2708-2726.

[17] B. Fehrensen, D. Luckhaus, M. Quack, Z. Physik. Chem. 1999, 209, 1.

[18] B. Fehrensen, D. Luckhaus, M. Quack, Chem. Phys. Lett. 1999, 300, 312.

[19] D. Luckhaus, J. Chem. Phys. 1997, 106, 8409.

[20] D. Luckhaus, J.L. Scott, C.F. Fleming, J. Chem. Phys. 1999, 110, 1533.

[21] R. Signorell, D. Luckhaus, J. Phys. Chem. A, submitted, 2001. 